DOI: 10.1002/ (adfm.201803629)

Article type: Full Paper

\title{
Anchor Groups for Graphene-Porphyrin Single-Molecule Transistors
}

Bart Limburg," James O. Thomas, " Gregory Holloway, Hatef Sadeghi, Sara Sangtarash, Ian Cheng-Yi Hou, Jonathan Cremers, Akimitsu Narita, Klaus Müllen, Colin J. Lambert, * G. Andrew D. Briggs, * Jan Mol, * and Harry L. Anderson*

\# These authors contributed equally

Dr. B. Limburg, Dr. J. O. Thomas, Dr. J. Cremers, Prof. H. L. Anderson

Department of Chemistry, University of Oxford, Chemistry Research Laboratory, Oxford OX1 3TA, UK

E-mail: harry.anderson@chem.ox.ac.uk

Dr. B. Limburg, Dr. J. O. Thomas, Dr. G. Holloway, Dr. J. Cremers, Prof. G. A. D. Briggs, Dr. J.

Mol

Department of Materials, University of Oxford, Parks Road, Oxford OX1 3PH, UK.

E-mail: andrew.briggs@materials.ox.ac.uk,jan.mol@materials.ox.ac.uk

Dr. H. Sadeghi, Dr. S. Sangtarash, Prof. C. J. Lambert

Quantum Technology Centre, Lancaster University, Lancaster LA1 4YB, UK.

E-mail: c.lambert@lancaster.ac.uk

I. C-.Y. Hou, Dr. A. Narita, Prof. K. Müllen

Max Planck Institute for Polymer Research, 55128 Mainz, Germany.

Keywords: single-molecules, graphene, porphyrins, charge transport, molecular electronics

Abstract: The effectiveness of five different anchor groups for non-covalent interfacing to graphene electrodes are compared. A family of six molecules is tested in single-molecule junctions: five consist of the same porphyrin core with different anchor groups, and the sixth is a reference molecule without anchor groups. The junction formation probability (JFP) has a strong dependence on the anchor group. Larger anchors give higher binding energies to the graphene surface, correlating with higher JFPs. The best anchor groups tested are 1,3,8-tridodecyloxypyrene and 2,5,8,11,14-pentadodecylhexa-peri-hexabenzocoronene, with JFPs of $36 \%$ and $38 \%$, respectively. Many junctions are tested at $77 \mathrm{~K}$ for each molecule by measuring source-drain current as a function of bias and gate voltage. For each compound, there is wide variation in the strength of the electronic coupling to the electrodes and in the location of Coulomb peaks. In most cases, this device-to-device variability makes it impossible to observe trends between the anchor and the charge-transport characteristics. Tetrabenzofluorene anchors, which are not $\pi$-conjugated with the 
porphyrin, exhibit different charge transport behavior to the other anchors tested, and they show multiple Coulomb peaks with characteristically small molecular electron-addition energies of $0.3-$ $0.7 \mathrm{eV}$, whereas the other compounds give single Coulomb peaks.

\section{Introduction}

The study of charge transport through individual molecules is important from both a technological and a fundamental scientific perspective. In general, the flow of charge through a molecule can only be studied when the molecule is functionalized with suitable anchor groups and connected to electrodes. Charge transport is controlled by the entire electrode/molecule/electrode architecture. Energy barriers between the anchor group and the electrodes lead to the formation of tunnel junctions. Significant variations in conductance between tunnel junctions containing the same molecule can occur due to differences in the details of the molecule-electrode coupling, and the conformation of the molecule within the junction. ${ }^{[1]}$ These phenomena modify the level broadening $(\Gamma)$ due to the molecule-electrode coupling and lead to differences in the conductance $(G)$ through the junction. For transport through a single molecular orbital, the conductance is conveniently quantified using the Landauer-Büttiker model, as given in equations (1) and (2), ${ }^{[2]}$ where $G_{0}$ is the conductance quantum, $T(E)$ is the transmission function, $f(E)$ is the Fermi-Dirac function, $\Gamma_{\mathrm{S}}$ and $\Gamma_{\mathrm{D}}$ characterize the coupling to the source and drain electrodes, respectively, $\left(\Gamma=\Gamma_{\mathrm{S}}+\Gamma_{\mathrm{D}}\right)$ and $\varepsilon$ is the energy of the molecular level, which may be shifted slightly by coupling to the electrodes.

$$
\begin{aligned}
& G=G_{0} \int_{-\infty}^{\infty} d E T(E)\left(-\frac{\partial f\left(E-E_{F}\right)}{\partial E}\right) \\
& T(E)=\frac{4 \Gamma_{S} \Gamma_{D}}{(E-\varepsilon)^{2}+\Gamma^{2}}
\end{aligned}
$$

Gold electrodes have been widely used to study single-molecule charge transport in scanning tunneling microscopy break-junctions (STM-BJs) and mechanically controlled break-junctions (MC-BJs). However, gold atoms undergo stochastic rearrangements at room temperature, as well as electromigration, causing fluctuations in $\Gamma$ and $G .^{[3]}$ In conductance measurements through selfassembled monolayers, such variations are averaged out due to the large number of molecules 
performing electron transport. For single-molecule STM-BJs and MC-BJs, it is possible to measure thousands of junctions, so that statistical analysis can be used to extract parameters such as the most probable conductance of a molecular junction.

$s p^{2}$-Carbon electrodes, such as carbon nanotubes and graphene, ${ }^{[4]}$ are promising alternatives to metal break junctions. The mobility of carbon atoms in graphene is lower than that of gold, making it possible to study the physics of single-molecule devices at room temperature. ${ }^{[5]}$ The device architecture allows for fabrication on a silicon substrate, which can serve as a global back-gate. The gate electrode provides an additional parameter to tune the alignment of the energy-levels of the molecule with respect to the Fermi energy of the leads, which is difficult to achieve in gold BJs, making it possible to test charge transport through energy levels far from the Fermi energy. Covalent linkage of molecules to graphene edges can be achieved, for example through amide coupling. ${ }^{[5 c, 6]}$ Alternatively, the molecule-electrode coupling can be established by $\pi$-stacking of flat anchors onto graphene nanogaps (GNGs). This type of $\pi$-stacking could potentially reduce the variations in $\Gamma,{ }^{[1,7]}$ by averaging the interaction over a larger interfacial area, but in practice values of $\Gamma$ still vary over four orders of magnitude, ${ }^{[8]}$ and there are open questions about the influence of the edge chemistry of the graphene on parameters such as $\Gamma$. Previous investigations have used anthracene, ${ }^{[9]}$ tetrabenzofluorene $(\mathrm{TBF}),{ }^{[5 b]}$ pyrene $^{[8,10]}$ and $\mathrm{C}_{60}{ }^{[11]}$ as anchors. However there has been no systematic experimental study of the same redox-active molecule functionalized with a library of anchor groups. By analogy with such studies carried out in STM-BJ experiments, ${ }^{[12]}$ the investigation of a group of molecules that have been altered in a specific way will allow us to assess whether we are, in fact, measuring molecules spanning graphene electrodes, and whether this graphene-based platform is suitable for investigating structure-property relationships of single molecules.

One problem with fixed GNGs, compared with BJs, is that it is more difficult to generate enough devices to allow statistical analysis of the results. Many interesting phenomena such as room temperature single electron transistor (SET) behavior, ${ }^{[13]}$ magnetic anisotropy, ${ }^{[14]}$ and the 
influence of electron-phonon coupling on single molecules bridging nanogaps, ${ }^{[9,10 b]}$ have been observed but usually only in a few devices. The small numbers of working molecular devices are a result of the fabrication procedure required to generate the gaps and the low probabilities that GNGs display molecular signatures after deposition. Junction formation probabilities (JFP), i.e., how many devices show a molecular feature from the total number of devices, reported in recent literature range from $2-24 \%{ }^{[5 b, 8-11,13-15]}$ A comparison of the influence of the anchors on JFPs has not been possible based on these works as the central molecular unit, and the method for identifying a molecular device, vary from study to study.

We have undertaken the synthesis of a family of molecules (Scheme 1) that consist of the same redox-active porphyrin core: five functionalized with different $\pi$-anchors, and one reference molecule with no anchor groups. The charge transport properties of compounds 1-6 have been studied in order to address the following key questions: (a) What is the influence of the anchor group on the JFP, and which anchor group gives the highest JFP? (b) How does the strength of the coupling, $\Gamma$, change with the anchor groups? Recent theoretical work predicts differences in conductance between compounds $\mathbf{2}$ and $\mathbf{3}$ due to quantum interference effects, and a comparatively lower conductance for 4 due to the breaks in conjugation with the TBF anchor. ${ }^{[7 \mathrm{a}]}$ These predictions will be tested. Analogous experiments in STM-BJs exploring different anchor groups for binding to gold electrodes have been an important step in understanding charge transport through molecules with STM-BJ and MCBJ experiments. ${ }^{[12 \mathrm{a}]}$

Single-electron transistor (SET) behavior is observed after depositing molecules that weakly couple to the electrodes; some of the key concepts of SET behavior, such as the links between Coulomb blockade and the Landauer-Büttiker model, are described in the SI. The emergence of SET behavior after molecular deposition is often used as proof that a molecule is bridging the tunnel junction. As discussed below, it is crucial to characterize the gate-dependence of the tunnel junction prior to molecular deposition, because electroburned GNGs can display SET behavior due to the accidental formation of graphene dots. ${ }^{[16]}$ Further methods to identify the presence of single 
molecules bridging GNGs include the assignment of characteristic vibrational modes of the molecules, ${ }^{[9,10 b]}$ or the response of paramagnetic molecules to a magnetic field. ${ }^{[14]}$ Both of these methods require stability diagrams, i.e., three-dimensional plots of source-drain current $\left(I_{s d}\right)$ as a function of gate voltage $\left(V_{g}\right)$ and source-drain voltage $\left(V_{s d}\right)$, to be measured at temperatures of a few Kelvin or below, and are experimentally time-consuming. The spacings between the energy levels controlling charge transport through single-molecule junctions are typically much greater than $k_{\mathrm{B}} T$ under ambient conditions (where $k_{\mathrm{B}}$ is the Boltzmann constant and $T$ is temperature), meaning that SET behavior can be observed for molecules spanning GNGs even at room temperature. ${ }^{[5]}$ However, in this study we chose to work at an intermediate temperature of $77 \mathrm{~K}$ in order to improve the quality of the data. Therefore a third question we address is: (c) Can we identify types of charge-transport behavior that are uniquely characteristic of single-molecule junctions by carrying out measurements at $77 \mathrm{~K}$ ?

\section{Results and Discussion}

\subsection{Molecular design and synthesis.}

Five zinc porphyrin compounds were synthesized by bromination of porphyrin $\mathbf{1}$, followed by Sonogashira coupling with various anchor groups, $\mathrm{R}-\mathrm{C} \equiv \mathrm{C}-\mathrm{H}$ (Scheme 1). Compounds $\mathbf{2}-\mathbf{6}$ contain flat aromatic systems that bind non-covalently to graphene. ${ }^{[17]}$ The anchors consist of varying numbers of $\pi$-electrons, which alter the electronic coupling to the graphene, and compounds $\mathbf{5}$ and $\mathbf{6}$ additionally contain alkyl chains which increase the binding energy to graphene. ${ }^{[18]}$ The central porphyrin moiety contains bulky 3,5-bis(trihexylsilyl)phenyl side-groups, which enhance the solubility of the compounds, and prevent $\pi$-stacking. ${ }^{[5 b]}$ Porphyrin $\mathbf{1}$ is also tested as a reference molecule with no anchor group. The attachment of anchor groups modifies the frontier orbitals of the $\pi$-system, as well as influencing the JFP. ${ }^{[12 b]} \mathrm{UV}$-vis absorption spectroscopy and electrochemistry (see SI) indicate that the molecular levels of compounds 1-6 are shifted depending on the anchoring group, and therefore we also expect the molecular energy levels to shift with respect to the Fermi energy of the graphene electrodes $\left(E-E_{\mathrm{F}}\right)$. 


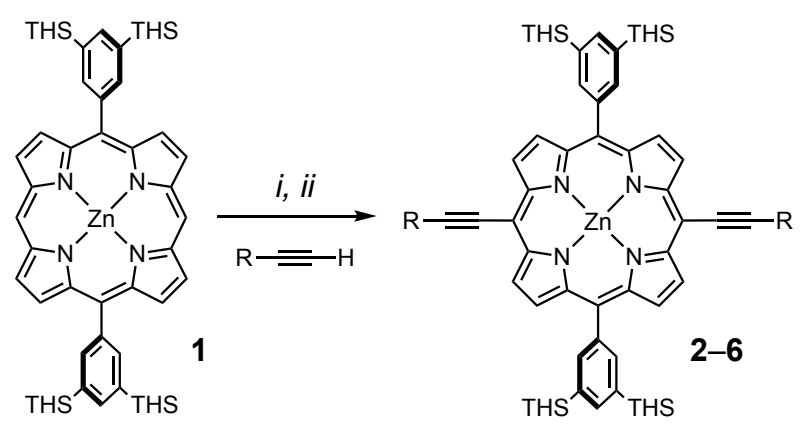

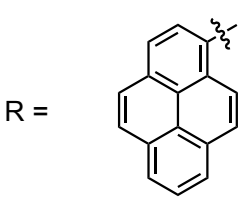

$2,52 \%$

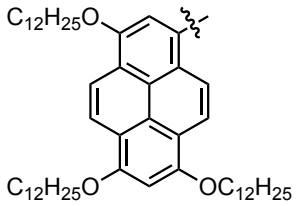

5, $25 \%$

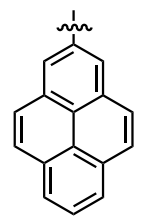

$3,32 \%$

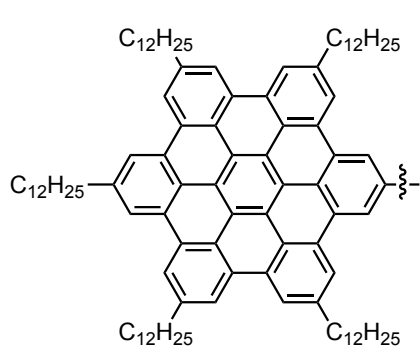

6, $40 \%$

Scheme 1. Synthesis of zinc porphyrins 2-6 from 1. i. $N$-Bromosuccinimide (2.1 eq.), $\mathrm{CHCl}_{3}$, pyridine, $20^{\circ} \mathrm{C}, 98 \% .{ }^{[19]} i i$. Sonogashira coupling conditions with $\mathrm{RC} \equiv \mathrm{CH}, 25-52 \%$ see SI. Yields are shown for the synthesis of 2-6 from the dibromo-porphyrin intermediate. THS $=$ trihexylsilyl.

\subsection{Fabrication.}

GNGs were fabricated by feedback-controlled electroburning of graphene nanoribbons containing a bowtie-shaped constriction contacted by gold electrodes, see Figure 1a. ${ }^{[13,20]}$ These constrictions were fabricated as follows. First, an array of $37 \times 23$ pairs of gold electrodes was patterned by

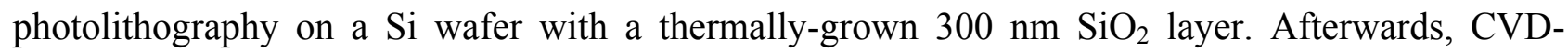
grown graphene was transferred from a copper substrate onto the chip and then patterned into the bowtie-shaped nanoribbons by electron-beam lithography. ${ }^{[20 c]}$ In this way, 851 devices were patterned on a $1 \times 1 \mathrm{~cm}$ chip and were electroburned over 24 hours on an automated probe-station. Figure $1 \mathrm{~b}$ shows a typical example of electroburning traces leading to a nanosized gap between the graphene electrodes. ${ }^{[13,20 c, 21]}$ The voltage across the graphene constriction is ramped up at a rate of 
5-8 $\mathrm{V} \mathrm{s}^{-1}$ up to a maximum of $10 \mathrm{~V}$, while the current is continuously monitored. If a rapid decrease in conductance is observed, the voltage is ramped back to $0 \mathrm{~V}$ at a rate of $2300-5000 \mathrm{~V} \mathrm{~s}^{-}$ 1. The process is repeated until the resistance of the junction exceeds $500 \mathrm{M} \Omega$. The red arrow in Figure $1 \mathrm{~b}$ indicates negative differential conductance present in the first electroburning trace. This decrease in conductance is attributed to thermal cleaning of the surface from residual contamination (as seen by AFM, Figure 1f; see SI for further images). ${ }^{[2]}$ The point indicated by the black arrow is the rapid decrease in conductance due to the electroburning process. In contrast to the electromigration process used to fabricate gold break-junctions, the formation of these nanogaps is not caused by migration of the edge atoms, but instead by sublimation of the graphene, if electroburning is carried out under vacuum, or by oxidation to generate $\mathrm{CO}_{2}$, if it is performed in air. $^{[20 \mathrm{a}]}$ A resistance measurement after the final green trace indicated that the electroburning process was complete, and produced the $I V$ trace in Figure 1c. A fit to the Simmons model including an asymmetric barrier ${ }^{[13,21,23]}$ (see SI) for the $I V$ trace of this particular device (Figure 1c) gave a gap width of $1.2 \mathrm{~nm}$, which is typical for GNGs electroburned in this way. The variation in the final resistance of devices that did (blue) or did not (orange) fit to the Simmons model is shown in Figure 1d, and the corresponding gap-widths of the fitted electroburned devices is shown in Figure 1e. Of the total number of devices, $64 \%$ could be fitted to the Simmons model $\left(R^{2}>\right.$ $0.95) ;{ }^{[13,21,23]}$ the mean gap width was $1.7 \mathrm{~nm}$ with a standard deviation of $0.6 \mathrm{~nm}$. The resistance of these devices had a narrow lognormal distribution centered on $1.1 \mathrm{G} \Omega$ (Figure 1d, blue histogram). The other devices either could not be electroburned and remained in the $\mathrm{k} \Omega$ range of resistance due to short-circuits with residual contamination, or broke during the process (Figure 1d, orange histogram). Figure 1f shows a phase-contrast AFM image of a correctly electroburned GNG. These AFM images reveal that particles of residual resist are removed from the graphene in a circular area around the nanojunction by the heating in this area during the electroburning process. ${ }^{[22]}$ By taking a histogram of the height of the surface, two clear peaks are distinguished: the planes of the $\mathrm{SiO}_{2}$ substrate and the graphene layer. Fitting each peak to a Gaussian function 
indicates that the graphene is $1.8 \mathrm{~nm}$ above the level of the $\mathrm{SiO}_{2}$ surface, which is a typical value for devices fabricated in this way. ${ }^{[24]}$

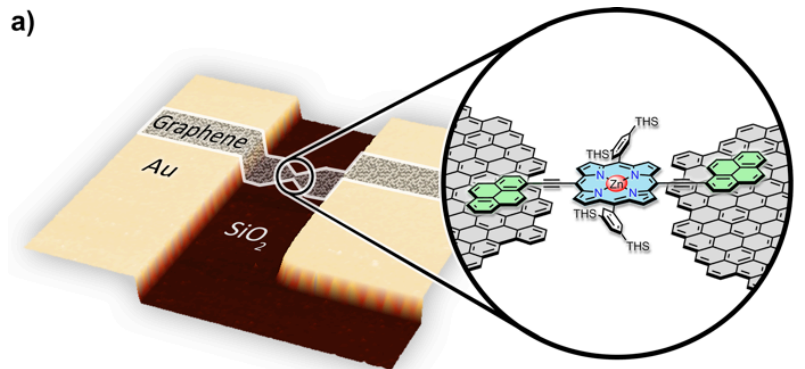

b)

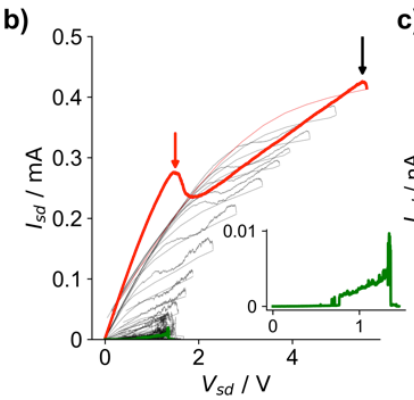

d)

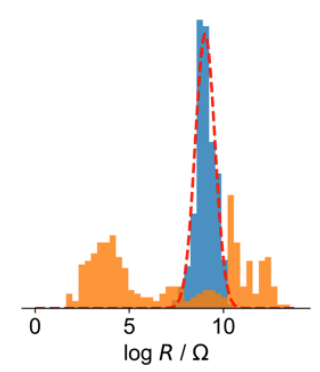

f)

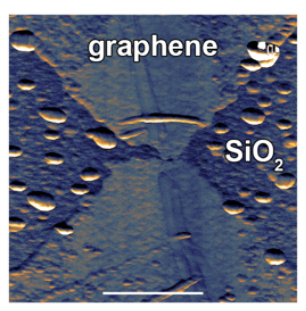

c)

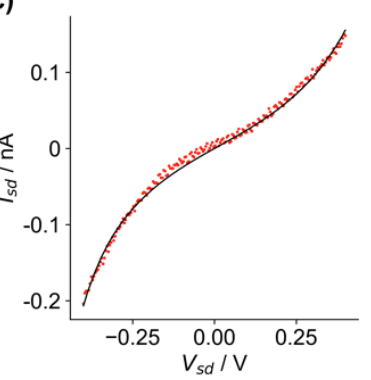

e)

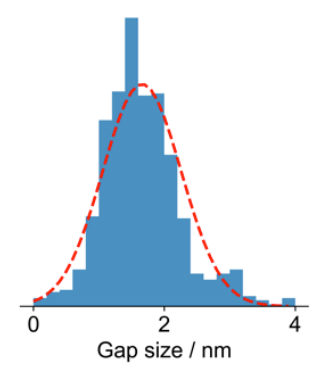

g)

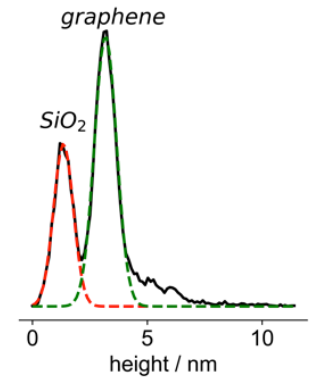

Figure 1. a) Schematic of our device architecture. The graphene is patterned into a bowtie-shape and electroburned to form a nanogap upon which molecules are deposited. b) Typical feedbackcontrolled electroburning traces. The first and last traces are indicated in red and green, respectively. Inset: The last electroburning trace. c) typical $I V$ trace after electroburning (red circles), and the corresponding fit to the asymmetric Simmons model (black solid line, prefactor: $0.155 \mathrm{nA}, \phi_{\mathrm{L}}: 0.30 \mathrm{eV}, \phi_{\mathrm{R}}: 0.57 \mathrm{eV}$, gap size: $1.17 \mathrm{~nm}$ ). d) Histogram of the resistance obtained from a linear fit of the $I V$ traces of all electroburned devices on a chip for devices that gave a bad fit (orange), or a good fit (blue) and the corresponding lognormal distribution fit (red dashed line). e) Histogram of the gap size obtained from fitting the $I V$ traces of all electroburned devices on a chip to the asymmetric Simmons model (see SI) and the corresponding normal distribution fit (red dashed line). f) False-color phase AFM image of an electroburned GNG. The scale bar is $250 \mathrm{~nm}$. g) Histogram of the heights of an AFM image of a GNG and fits of the $\mathrm{SiO}_{2}$ plane (red) and graphene plane (green). The graphene is $1.8 \mathrm{~nm}$ above the $\mathrm{SiO}_{2}$ plane. 


\subsection{Charge stability diagrams.}

After electroburning, each chip was cut into nine evenly sized pieces and 19 devices with good fits and resistances between $0.5-5 \mathrm{G} \Omega$ on each piece were wire-bonded to a chip carrier. Therefore, 171 devices out of the 851 devices per chip were used for further experiments. The bare GNGs were cooled to $77 \mathrm{~K}$ under vacuum and stability diagrams were recorded with a gate voltage $\left(V_{g}\right)$ range of $\pm 80 \mathrm{~V}$, and a bias voltage $\left(V_{s d}\right)$ range of $\pm 0.4 \mathrm{~V}$. The electroburning process produces tunneling nanogaps such as seen in Figure 2a, where the only significant feature is an increase in conductance of the junction at negative gate voltages. We assign this feature to an increase in charge carrier concentration due to gating the graphene further from the Dirac point. Inevitably, the process also produces devices with graphene quantum dots, as seen in Figure 2b. Such dots exhibit SET behavior, showing resonant transport regimes, as well as Coulomb-blocked regions. The zero-bias gate trace (Figure $2 \mathrm{~b}$ right) shows Coulomb peaks. In addition, some devices break during the wirebonding, pumping or cooling to $77 \mathrm{~K}$, showing no current above the noise-floor. Lastly, many devices do not reveal clear quantum dot features, but show unassigned structure in the stability diagram besides the Dirac-point feature (Figure 2c). 

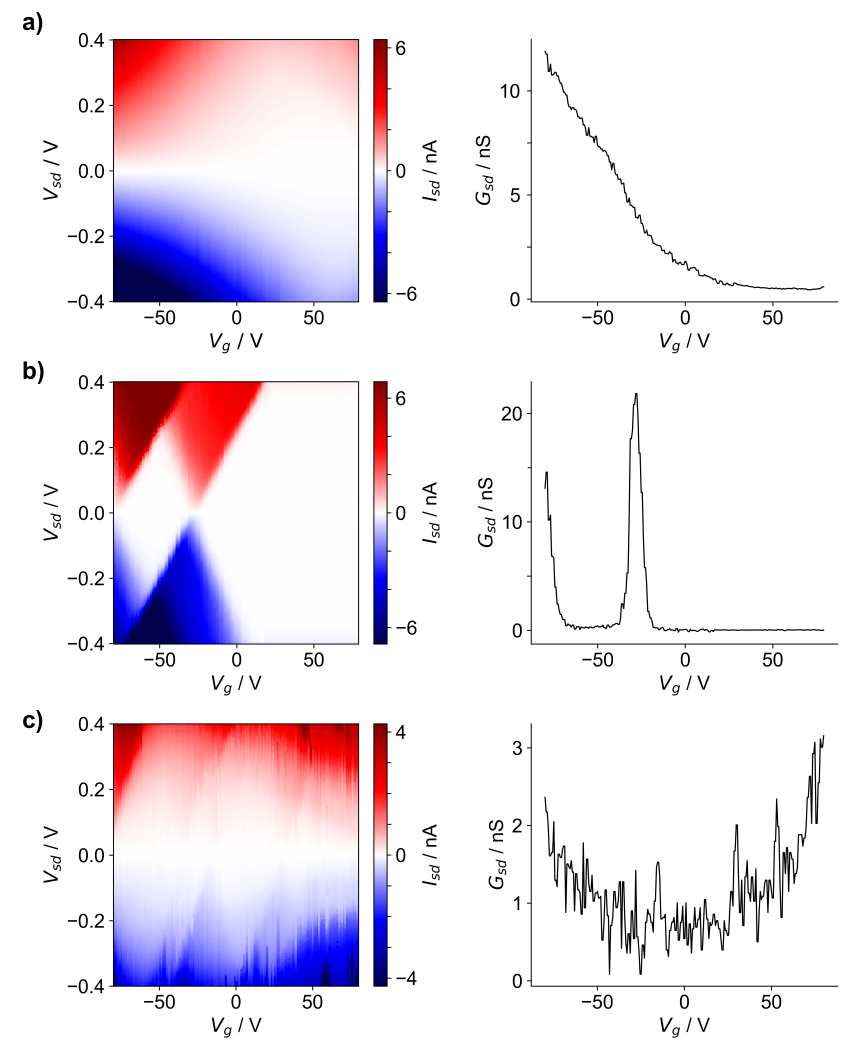

Figure 2. Left: Stability diagrams for three devices after electroburning, measured at $77 \mathrm{~K}$ under vacuum. Right: source-drain conductance at $0 \mathrm{~V}$ bias $v s$. gate voltage. a) A graphene nanogap without any quantum dot features, only an increase in conductance due to the Dirac point is visible at negative gate voltages. b) A graphene nanogap with clear quantum dot SET features leading to Coulomb blockade. A peak is visible on the $0 \mathrm{~V}$ bias gate trace due to resonant transport through the quantum dot. c) A graphene nanogap with unclear features that could not be assigned.

After recording the stability diagrams of the GNGs, a solution of one of the compounds 1-6 (2 $\mu \mathrm{L}, 3.0 \mu \mathrm{M}$ in toluene) was drop-cast onto each chip-piece and allowed to evaporate in air at room temperature. The devices were then cooled to $77 \mathrm{~K}$ under vacuum again and post-deposition stability diagrams were measured. The amount of compound roughly corresponds to a monolayer on the surface, and an AFM study of compound $\mathbf{2}$ showed that it forms a uniform layer and does not aggregate on graphene (see SI). To avoid unconscious bias in the analysis, we first categorized the stability diagrams according to the three types displayed in Figure 2 (empty, SET or unassigned) without knowing whether the stability diagram was recorded before or after deposition of a molecule. Afterwards, the stability diagrams categorized as 'empty' or as 'SET' (Figure 2a or b, respectively) were further analyzed by comparing the results before and after deposition, still without knowledge of which molecule was deposited. The results were categorized by looking for 
changes from an empty nanogap to a SET feature caused by the molecule (Figure 3, category A), or an empty nanogap that remained empty (Figure 3, category B). All other data that showed ambiguous features such as those seen in Figure 2c were discarded (category $\mathbf{C}$ ). It is necessary to go through this rigorous method in order to ensure that the features observed in the stability diagrams are caused by the deposited molecule. Other methods, such as measuring zero bias and gate voltage conductance are insufficient, for two reasons: 1) the anchor groups provide weak coupling to the electrodes and therefore transport is dominated by single-electron transistor behavior, i.e., Coulomb blockade and resonant transport, with resonances occurring when the molecular levels enter the bias window. These resonances tend to occur far from zero gate voltage, ${ }^{[25]} 2$ ) the graphene nanogap size varies over a large range (see Figure 1e), which means that the background tunneling current varies over several orders of magnitude, obscuring any offresonant features of the molecule. The resistances of all the devices at zero bias and zero gate voltages before and after molecular deposition are shown in the SI.
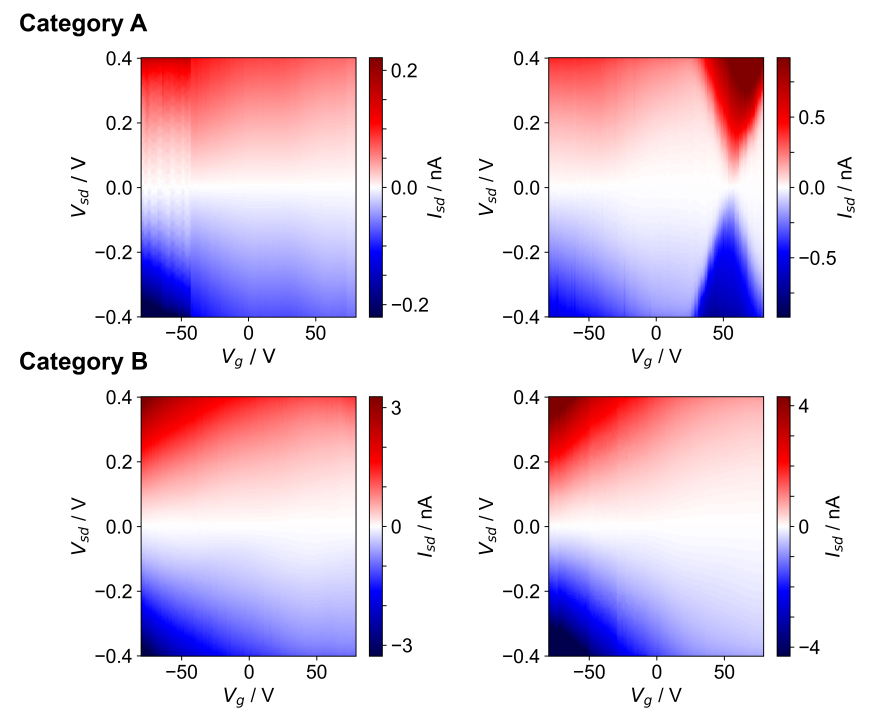

Figure 3. Comparison of the stability diagrams for two devices before and after molecular deposition measured at $77 \mathrm{~K}$ under vacuum. Category $\mathbf{A}$ : a molecular feature is present after deposition. Category B: no significant changes occurred after deposition.

\subsection{Statistics.}

During this study, 52 chip pieces were measured and analyzed, with 19 devices wire-bonded per chip piece, corresponding to a total of 988 devices. Of these devices, 221 had leakage currents from the gate electrode, prohibiting the measurements. A further 284 devices did not provide any 
measurable current above the noise-floor and were therefore categorized as broken. Of the remaining 483 devices, 26 were of category A (empty/SET; see SI for all stability diagrams), and 101 were of category $\mathbf{B}$ (empty/empty). The remaining ambiguous data (category $\mathbf{C}, 356$ devices that contained SET features prior to molecular deposition, or could not be clearly assigned) were discarded. Next, the well-behaved category A devices were split into their molecular types. Junction formation probabilities (JFPs) are calculated for each molecule by dividing the number of category A devices by the sum of category $\mathbf{A}$ and $\mathbf{B}$. These values are reported in Table 1. Our definition of the JFP ignores the devices that show ambiguous features (category $\mathbf{C}$ ), because we cannot know whether features present in such devices are caused by the molecule. ${ }^{[26]}$

Table 1. Statistics of junction formation probability (JFP) of all measured devices per compound 16.

\begin{tabular}{|c|c|c|c|c|c|c|}
\hline Compound & 1 & 2 & 3 & 4 & 5 & 6 \\
\hline Total number of devices measured ${ }^{\text {a) }}$ & 133 & 133 & 209 & 171 & 171 & 171 \\
\hline Category $\mathbf{A}$ devices (molecular junction) & 0 & 3 & 3 & 4 & 8 & 8 \\
\hline Category B devices (empty junction) & 20 & 20 & 17 & 17 & 14 & 13 \\
\hline Junction formation probability (JFP) & $0 \%$ & $13 \%$ & $15 \%$ & $19 \%$ & $36 \%$ & $38 \%$ \\
\hline Anchor group binding energy $(e V)^{b)}$ & & -0.84 & -0.84 & -1.26 & -3.23 & -5.53 \\
\hline$p$-value (compared with compound 1$)^{c)}$ & & 0.22 & 0.20 & 0.13 & 0.023 & 0.021 \\
\hline Device yield ${ }^{d)}$ & $0 \%$ & $2.3 \%$ & $1.4 \%$ & $2.3 \%$ & $4.7 \%$ & $4.7 \%$ \\
\hline
\end{tabular}

\footnotetext{
a) The total number of devices includes all the devices that were wire-bonded and cooled to $77 \mathrm{~K}$. Of these, the number of category $\mathbf{C}$ devices per molecule were: 1: 30, 2: 41, 3: 89, 4: 77, 5: 54, 6: 65 . The remaining devices (those that are not categorized) were broken. b) Binding energies were calculated by SIESTA ${ }^{[27]}$ implementation of DFT (see SI). ${ }^{\text {c) }}$ The $p$-value is a comparison to compound $\mathbf{1}$ as explained in this text and SI. ${ }^{\text {d) }}$ The device yield is defined as the number of category A devices divided by the total number of devices measured.
}

The data in Table 1 indicate that the JFP depends on the anchor group. The probability that a category A device will be made by drop casting compound $\mathbf{1}$ is very low, intermediate for compounds 2-4, whereas for compounds 5 and 6 it reaches values of $36 \%$ and $38 \%$, respectively. This is consistent with our binding energy calculations of the molecular structures (see Table S2 and Figure S6 in Section 6 in the SI) where 5 and 6 have larger binding energy to graphene. Intuitively, this result is significant, but a statistical analysis is required in order to exclude the possibility that the observed results are produced merely by chance. Therefore, a statistical test to compare results between two sets of data was used. This test provides us with a $p$-value for the null- 
hypothesis: "All sets of data originate from the same statistical process". If this $p$-value is lower than 0.05 , we may discard the null-hypothesis. The statistical method used is described in the SI. When comparing the JFP of molecule $\mathbf{1}$ to $\mathbf{6}$, the corresponding $p$-value is 0.021 , which allows us to discard the null-hypothesis in favor of concluding that the JFP is different. A $p$-value of 0.023 is obtained by comparing 1 and 5. While the results for compounds $2-\mathbf{4}$ are not statistically relevant ( $p$ $>0.05$ ) compared to the reference compound $\mathbf{1}$, they fit the trend that large $\pi$-systems increase the JFP, and that additional alkyl tails on the $\pi$-systems act as a glue that helps to stick the $\pi$-system to the graphene. As such, the results in Table 1 are intuitively understood as the increase in binding energy of the anchor to graphene correlates with an increase in JFP. Sufficient binding energy of the anchor to the graphene is likely required to displace adsorbed hydrocarbons, water, and other atmospheric contaminants, ${ }^{[28]}$ as well as potential geometric changes (such as rotation of the aryl groups) necessary for adsorption, but increasing the binding energy further (comparing 5 with 6) does not improve the JFP.

\subsection{Molecular conductance.}

Next, we evaluated the reproducibility of the electronic coupling of the molecule to the electrodes. Stability diagrams were measured at $77 \mathrm{~K}$. At this temperature, $k_{\mathrm{B}} T>>\Gamma$ and the Coulomb peak in gate voltage traces is thermally broadened, and can be described by the function: ${ }^{[29]}$

$$
G_{\mathrm{sd}}\left(V_{\mathrm{g}}\right)=G_{\max } \cosh ^{-2}\left(\frac{\alpha e\left(V_{\mathrm{g}}-V_{\mathrm{c}}\right)}{2 k_{\mathrm{B}} T}\right)
$$

where $G_{\max }$ is the maximum conductance, $e$ is the elementary charge, $\alpha$ is the lever arm (i.e., the ratio of capacitance of the gate electrode to the total capacitance, $\left.C_{\mathrm{g}} / C_{\mathrm{tot}}\right)$, and $V_{\mathrm{c}}$ is the gate voltage at the center of the Coulomb peak. From equation (3), we can obtain the coupling to the electrodes $(\Gamma)$, and the energy of the resonant level relative to the Fermi level according to the relations $^{[8]}$ 


$$
\Gamma=4 k_{\mathrm{B}} T \frac{\hbar}{e^{2}} G_{\max }
$$

$$
E-E_{\mathrm{F}}=\alpha e V_{\mathrm{c}}
$$

where $\hbar$ is the reduced Planck's constant and $E_{\mathrm{F}}$ is the energy of the Fermi level. Because of the high temperature and weak coupling $\left(k_{\mathrm{B}} T>>\Gamma\right)$, we can safely assume that there is no significant contribution of lifetime broadening to the line-shape of the Coulomb peak. Figure 4a, b and c depict the fits for three different devices fabricated using compounds $\mathbf{6 , 5}$ and $\mathbf{3}$, respectively, as examples. The gate traces were background-corrected for the Dirac point feature by subtracting an exponential decrease function and fitted to equation (3). The stability diagrams belonging to category A were described very well by equation (3), and the coupling to the leads $(\Gamma)$ and the lever arm $(\alpha)$ could be obtained for most (19) of the devices. The values of $\Gamma$ exhibit a large spread in values. For example, the eight junctions functionalized with molecule 5 exhibit $\Gamma$ values in the range: $4.1 \times 10^{-8}-5.4 \times$ $10^{-4} \mathrm{eV}$, and for molecule 6 the values of $\Gamma$ are in the range $1.9 \times 10^{-7}-3.5 \times 10^{-4} \mathrm{eV}$. The spread of these values over four orders of magnitude is consistent with the range extracted using a fullerene derivative with a pyrene anchor on graphene in a previous study. ${ }^{[8]}$ Values of $\Gamma$ and $E-E_{\mathrm{F}}$ were extracted by fitting equation (3) to all the stability diagrams that show Coulomb blockade features: both those of the molecular devices and those of devices showing SET behavior before molecular deposition due to graphene quantum dots (for example: Figure 2b). These data, plotted in Figure 5, show that, with a limited number of devices, the different compounds 2-6 do not give a reproducible coupling to the leads. For compound 4, two Coulomb peaks were observed in three out the four molecular devices, and therefore seven values of $\Gamma$ and $E-E_{\mathrm{F}}$ could be extracted and plotted. For others, some fits were of poor quality and as such some points were left out. If charge transport is described only by $\Gamma$ and $E-E_{\mathrm{F}}$, these molecules are indistinguishable from the graphene quantum dots that are occasionally present before molecular deposition. There are several potential reasons for the high variability, such as the conformation of the molecule in the nanogap, ${ }^{[7 \mathrm{a}]}$ its 
rotation with respect to the axis normal to the graphene plane, and the graphene edge chemistry. In our experiments, we do not control the orientation of the molecule, a small translation or rotation of the molecule with respect to the graphene will change the overlap of the $\pi$-anchors with the graphene and could significantly affect $\Gamma$. Similarly, as we cannot control the atomic details of the graphene junction formed during the electroburning process, there can be variation in the edge chemistry that will affect the transport. ${ }^{[30]}$ However, for gold break-junction experiments, the conductances of thousands of successful junctions are plotted on a log scale to obtain a most probable molecular conductance. The standard deviation in the conductance of these BJ experiments can be several orders of magnitude, which is similar to the deviation in Figure 5.
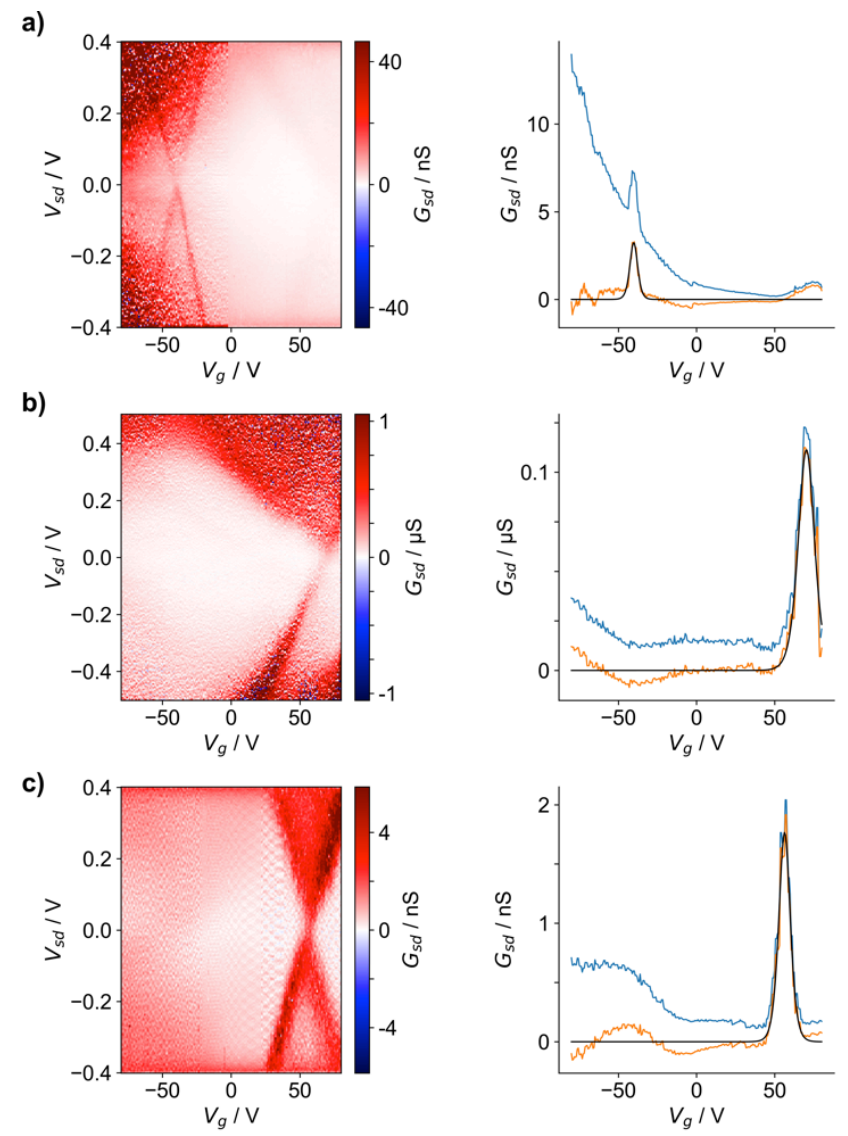

Figure 4. Examples of conductance stability diagrams measured at $77 \mathrm{~K}$ under vacuum (left) and zero bias conductance gate traces (right) of three devices. The gate traces (blue) were background corrected using an exponential decrease function and the background corrected trace (orange) was fitted to equation (1) (black). a) Compound $\mathbf{6}, \alpha=4.0 \times 10^{-3}, \Gamma=1.42 \mu \mathrm{eV}, \mathrm{b}$ ) compound $\mathbf{5}, \alpha=1.9$ $\times 10^{-3}, \Gamma=48.6 \mu \mathrm{eV}, \mathrm{c}$ ) compound $3, \alpha=2.8 \times 10^{-3}, \Gamma=0.77 \mu \mathrm{eV}$. 


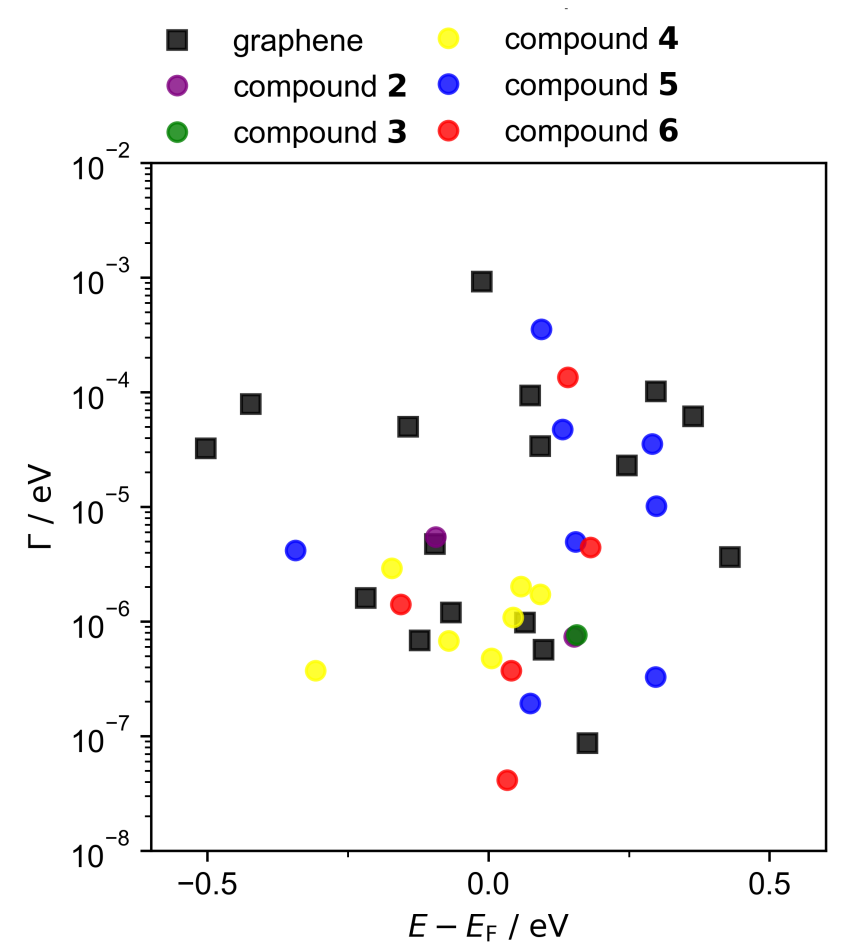

Figure 5. Scatterplot of the coupling to the leads $(\Gamma) v s$. the energy of the resonant level relative to the Fermi level $\left(E-E_{\mathrm{F}}\right)$. Black squares: graphene quantum dots. Colored circles: molecules. 2: purple, 3: green, 4: yellow, 5: blue, 6: red.

Additional parameters can be extracted from stability diagrams, such as addition energies of the molecule within the junction. The addition energy, $E_{\text {add }}$, is the difference in potential between consecutive oxidation/reduction states of the molecule and it roughly corresponds to the gap between redox states measured by cyclic voltammetry. For compounds 2, 3, 5 and $\mathbf{6}$ only one Coulomb peak is observed in a gate trace, and no closing diamonds are present in the experimental window that we are able to access with our device geometry. These molecules have a HOMOLUMO gap of $\sim 2 \mathrm{eV}$ that defines a large energy-gap between the first oxidation and first reduction potential of the neutral compound (see SI). The values of $\alpha$, calculated by fitting the gate peaks to equation (3) are in the range $1.2 \times 10^{-3}-6.5 \times 10^{-3}$, with a mean of $3.0 \times 10^{-3}$. These low values for $\alpha$, combined with a gate voltage limit of $\pm 80 V_{\mathrm{g}}$ (to avoid large gate leakage currents or breakdown of the dielectric) means the energy levels of the molecules are shifted over a ranges between $0.20-$ $1.0 \mathrm{eV}$ within a stability diagram, with an mean accessible range of $0.48 \mathrm{eV}$, for each stability diagram. These ranges are small compared to the addition energies of the molecules $\mathbf{2}, \mathbf{3}, \mathbf{5}$ and $\mathbf{6}$, and therefore stability diagrams of these molecules present a single Coulomb peak. In contrast, we 
observed multiple Coulomb peaks in the gate traces for molecules of compound 4. Addition energies were extracted for three out of four molecular devices. The addition energies are all in the range of $0.3-0.7 \mathrm{eV}$ for the stability diagrams are shown in Figure 6 . This value of the addition energy agrees with a previous study of the charge transport properties of compound $\mathbf{4}$ in GNGs, ${ }^{[5 b]}$ but it is much less than the HOMO-LUMO gap from cyclic voltammetry (see SI Section 3). It is not clear why compound $\mathbf{4}$ exhibits such small addition energies, but this behavior may be related to the break in $\pi$-conjugation between the anchor groups and the porphyrin core. The molecule can therefore be separated into anchor-states and porphyrin-states which will couple differently to the electrodes. Theoretical calculations have predicted that molecule $\mathbf{4}$ has two closely spaced peaks in the transmission function in the HOMO-dominated transport region, which could lead to the observation of Coulomb diamonds with a small $E_{\text {add }}$ that is mostly determined by the charging energy rather than the level spacing. ${ }^{[7 \mathrm{a}]}$

Currently, with the variability in $\Gamma$ and the inability to measure $E_{\text {add }}$ for compounds $\mathbf{2}, \mathbf{3}, \mathbf{5}$ and 6, the most reliable way to identify a molecular junction without moving to temperatures of a few Kelvin is to take a full stability diagram before and after molecular deposition. In many of our cases the resonant transport portion of the stability diagram does not overlap with a $I-V_{\text {sd }}$ trace at zero gate voltage. Thus, an $I-V_{\text {sd }}$ trace before and after deposition is not a suitable way to identify junctions that have been functionalized by molecules, and it is necessary to measure the gate dependence of each device before and after.

The differences in the JFP for different molecular structures together with the observation of multiple Coulomb peaks only for compound 4 provides firm evidence that the observed SET effects are caused by the presence of a molecule in the gap, and more importantly that the device properties depend on the choice of molecule. The variability in coupling strength and energy-level alignment makes it difficult to identify molecular structure-property relationships. This variability may be intrinsic to graphene-based molecular junctions. In contrast to the well-developed gold breakjunction experiments, it is not possible to obtain thousands of single-molecule conductance 
measurements with our devices. Therefore further development in the field of graphene-based single molecule junctions requires focus of the research community on issues of reproducibility and acquisition of sufficient data to test for the statistical significance of apparent trends. An advantage of the use of an electrostatic gate is that we have access to additional spectroscopic information on our single-molecule junctions. Our results show that reproducible addition energies are observed as a characteristic molecular feature, even with widely varying coupling strengths and energy-level alignment. This work highlights the importance of anchor groups and demonstrates a method to fabricate graphene single-molecule transistors with a high device yield.
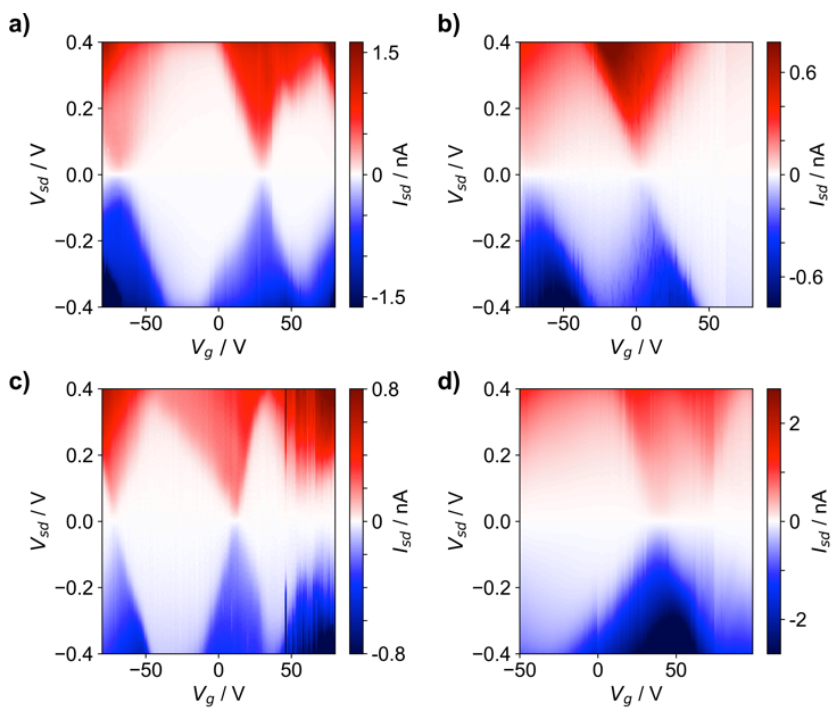

Figure 6. The four category A stability diagrams measured at $77 \mathrm{~K}$ under vacuum for molecule 4. For a) and c) two addition energies, $(0.53 \mathrm{eV}, 0.31 \mathrm{eV})$ and $(0.68 \mathrm{eV}, 0.46 \mathrm{eV})$ respectively, were extracted. The addition energy for $\mathrm{b}$ ) is $0.45 \mathrm{eV}$. For the device shown in $\mathrm{d} \alpha$ is low and the range over which the energy levels can be shifted is only $0.22 \mathrm{eV}$, and therefore only one Coulomb peak was observed.

\section{Conclusions}

In conclusion, we report the first systematic study of a family of molecules deposited on GNGs to further our understanding of single-molecule charge transport measurements using $\pi$-stacking to connect the molecule with graphene electrodes. In response to the questions posed in the introduction, our results demonstrate that anchor groups do have a strong effect on the JFPs of molecular devices. The JFP correlates with an increase in the binding energy of the anchor to the graphene surface. However, we have found that, with our limited number of devices, it is not 
possible to correlate changes in the anchor with changes in the electronic coupling to the leads, $\Gamma$. The data presented here corroborate previous results ${ }^{[8]}$ that show a spread of $\Gamma$ over four orders of magnitude. The theoretical prediction that a narrow distribution of $\Gamma$ could be expected for spatially extended anchor groups $^{[7 b]}$ is not supported by our experimental data, and the prediction of variation in $\Gamma$ between different anchors ${ }^{[7 \mathrm{a}]}$ could not be tested due to the high device-to-device variability. Further experiments are needed to understand the origins of this variability so that it can be mitigated; possible causes include different orientations of the molecule across the gap and variation in the edge chemistry of the graphene. The reproducibility of fabrication of empty GNGs (devices only showing a Dirac point feature) also needs to be improved, to make the device yield closer to the JFP (i.e., to reduce the number of category $\mathbf{C}$ and broken devices). It will be interesting to explore whether changing the functional groups on the edge of the graphene reduces device-todevice variability and increases the device yield.

Our results show that the electroburning process can lead to residual graphene quantum dots in GNGs, and, if the junctions are described only in terms of $\Gamma$ and $E-E_{\mathrm{F}}$, these quantum dots cannot be distinguished from molecular features. However, we only observed the emergence of SET behavior in gaps that were originally empty when the GNGs were treated with solutions of compound 2-6, with anchor groups, not with compound $\mathbf{1}$ without anchors. Therefore, we conclude that molecular features can be identified when SET behavior emerges after molecular deposition. JFPs for molecules 5 and $\mathbf{6}$ are the highest reported in the literature so far, at $36 \%$ and $38 \%$. The knowledge that certain anchor groups, specifically those functionalized with long alkyl chains, lead to high JFPs will guide future molecular design and facilitate the study of single-molecule transistors.

The stability diagrams of compound 4 exhibit features from more than one redox process, enabling the addition energy ( $\left.E_{\text {add }}\right)$ to be estimated, whereas this is not possible for the other compounds because they never show more than one redox process in the accessible range of gate voltages. Thus we demonstrate that it is possible to extract molecular features from graphene-based 
single molecule transistors at $77 \mathrm{~K}$, provided that these features are unaffected by the variations in $\Gamma$ and provided that stability diagrams are compared before and after molecular deposition. However, measurements at $77 \mathrm{~K}$ are less informative than low temperature studies $(T \leq 4 \mathrm{~K})$ in which charge transport features specific to single molecules become apparent, such as the electron-phonon coupling and response to a magnetic field. The large binding energies of the new anchors in compounds $\mathbf{5}$ and $\mathbf{6}$, and the subsequent impact on our device yields, suggest that these anchors could be valuable in other fields such as bioelectronics and materials science, which also make use of non-covalent molecular anchoring to graphene via $\pi$-stacking for device fabrication. ${ }^{[31]}$

\section{Experimental Section}

Substrate fabrication. Silicon wafers $(1 \times 1 \mathrm{~cm})$ with thermally grown $\mathrm{SiO}_{2}(300 \mathrm{~nm})$ were sonicated for $5 \mathrm{~min}$ in acetone, and subsequently in iso-propanol (IPA). Onto the wafer was spincoated PMGI SF6, and the wafer baked at $150{ }^{\circ} \mathrm{C}$ for $90 \mathrm{~s}$. Subsequently, S1805 was spin-coated on top of the PMGI SF6 layer and the wafer baked at $120^{\circ} \mathrm{C}$ for $90 \mathrm{~s}$. The electrode design was patterned using photolithography and developed using MF319 for 45 s and washed with $\mathrm{H}_{2} \mathrm{O}$. Afterwards, $10 \mathrm{~nm}$ of $\mathrm{Cr}$ and $80 \mathrm{~nm}$ of $\mathrm{Au}$ were evaporated onto the wafer. Finally, lift-off was performed using Remover PG and the sample washed with IPA, acetone and IPA again.

Graphene transfer. CVD-grown single-layer graphene on copper (either purchased from Graphenea or synthesized in house) with a spin-coated PMMA layer (PMMA-graphene-copper) was cut into a $1 \times 1 \mathrm{~cm}$ piece. The copper was etched by floating the stack on an aqueous solution of ammonium persulfate $(3.6 \mathrm{~g}$ in $40 \mathrm{~mL}$ ) for $20 \mathrm{~h}$. Afterwards, the PMMA-graphene was transferred using a clean microscope glass to fresh $\mathrm{H}_{2} \mathrm{O}$ three times. The graphene was scooped up using the substrate, and carefully dipped halfway in IPA to remove air and $\mathrm{H}_{2} \mathrm{O}$ trapped under the graphene. Afterwards, the sample was reversed and the other half was dipped into IPA. The sample was left to dry for at least $1 \mathrm{~h}$ after which it was baked for $1 \mathrm{~h}$ at $180{ }^{\circ} \mathrm{C}$. After cooling, the sample was submerged in acetone at $50{ }^{\circ} \mathrm{C}$ for at least $3 \mathrm{~h}$ to remove the PMMA. 
Graphene patterning. The sample containing graphene on top of gold electrodes was spin-coated with ma-N 2403 and then baked at $90{ }^{\circ} \mathrm{C}$ for $60 \mathrm{~s}$. Bowtie-shaped graphene was generated using electron beam lithography with a dose of $120 \mu \mathrm{C} \mathrm{cm}^{-2}$. The sample was developed using ma-D 525 for $60 \mathrm{~s}$ and washed with $\mathrm{H}_{2} \mathrm{O}$. The graphene at the unexposed areas was removed using oxygen plasma (45 s). The photoresist was removed by flowing mr-Rem 660 over the sample which was submerged in mr-Rem 660. The sample was transferred to a second solution of mr-Rem 660 in which it was swirled for $3 \mathrm{~min}$. Finally, the sample was washed with acetone and IPA. The graphene nanogaps were electroburned as described in the main text.

Measurement setup. Charge stability diagrams were measured in a 'dip-stick' setup. The sample of graphene nanogaps was wire-bonded in a chip-carrier and placed in a capped metal tube. After evacuating the tube to a base pressure of $<2 \times 10^{-3}$ mbar, it was placed in a Dewar filled with liquid nitrogen. After waiting for the temperature of the sample to settle (approximately $1 \mathrm{~h}$ ), measurements were started. As a source-drain voltage source, a HP33120A function generator was used, set to $1 \mathrm{~Hz}$ triangular wave with amplitude of 0.4 or $0.5 \mathrm{~V}$. The gate voltage was applied by a Keithley 2450 SourceMeter. The source-drain current was amplified by a Stanford Research Systems SR570 current amplifier automatically gained to the correct settings by the computer, and the data collected by a National Instruments BNC-2090A DAQ. Devices were consecutively connected / grounded automatically by a house-built automatic signal router. Due to the low-pass filter on the input of the current amplifier and the $1 \mathrm{~Hz}$ function generator, applied bias voltages may have been slightly off. As we do not use the bias voltages for any precise measurements, we have neglected this artifact and used the average current over the two directions of the triangular wave.

Computational methods. The optimized geometry and ground state Hamiltonian and overlap matrix elements of each structure studied in this paper was self-consistently obtained using the SIESTA ${ }^{\text {[27] }}$ implementation of density functional theory (DFT). SIESTA employs norm-conserving pseudopotentials to account for the core electrons and linear combinations of atomic orbitals $(L C A O)$ to 
construct the valence states. For binding energy calculations, the non-local van der Waals density functional $(v d W-D F)$ is used with $\mathrm{BH}$ parameterization and a double- $\zeta(D Z)$ basis set. The realspace grid is defined with an equivalent energy cut-off of 250 Ry. The geometry optimization for each structure is performed until the forces on each atom are smaller than $20 \mathrm{meV} \AA^{-1}$.

\section{Supporting Information}

Synthetic procedures, characterization data, UV-vis spectra, redox potentials, device fabrication and characterization, single electron transistor explanation, binding energy calculations, charge stability diagrams of molecular devices, addition energy characterization, $p$-values and junction resistance before/after comparison. The Supporting Information is available from the Wiley Online Library or from the author.

\section{Acknowledgements.}

This work was supported by the EPSRC (grants EP/N017188/1, EP/N014995/1 and EP/R029229/1) and partially by the Max Planck Society. H.S. acknowledges Leverhulme Early Career Fellowship no. ECF-2017-186. We thank Y. Sheng and J. H. Warner for providing some of the graphene used in these experiments.

Received: ((will be filled in by the editorial staff))

Revised: ((will be filled in by the editorial staff)) Published online: ((will be filled in by the editorial staff)) 
[1] a) J. Šebera, V. Kolivoška, M. Valášek, J. Gasior, R. Sokolová, G. Mészáros, W. Hong, M. Mayor, M. Hromadová, J. Phys. Chem. C 2017, 121, 12885-12894; b) X. Li, J. He, J. Hihath, B. Xu, S. M. Lindsay, N. Tao, J. Am. Chem. Soc. 2006, 128, 2135-2141; c) E. Lörtscher, Nat. Nanotechnol. 2013, 8, 381; d) H. Joshua, T. Nongjian, Semicond. Sci. Technol. 2014, 29, 054007; e) T. A. Su, M. Neupane, M. L. Steigerwald, L. Venkataraman, C. Nuckolls, Nat. Rev. Mater. 2016, 1, 16002.

[2] C. J. Lambert, Chem. Soc. Rev. 2015, 44, 875-888.

[3] G. K. Ramachandran, T. J. Hopson, A. M. Rawlett, L. A. Nagahara, A. Primak, S. M. Lindsay, Science 2003, 300, 1413.

[4] C. Jia, B. Ma, N. Xin, X. Guo, Acc. Chem. Res. 2015, 48, 2565-2575.

[5] a) M. L. Perrin, E. Burzuri, H. S. J. van der Zant, Chem. Soc. Rev. 2015, 44, 902-919; b) J. A. Mol, C. S. Lau, W. J. M. Lewis, H. Sadeghi, C. Roche, A. Cnossen, J. H. Warner, C. J. Lambert, H. L. Anderson, G. A. D. Briggs, Nanoscale 2015, 7, 13181-13185; c) Q. Xu, G. Scuri, C. Mathewson, P. Kim, C. Nuckolls, D. Bouilly, Nano Lett. 2017, 17, 5335-5341.

[6] C. Jia, A. Migliore, N. Xin, S. Huang, J. Wang, Q. Yang, S. Wang, H. Chen, D. Wang, B. Feng, Z. Liu, G. Zhang, D.-H. Qu, H. Tian, M. A. Ratner, H. Q. Xu, A. Nitzan, X. Guo, Science 2016, 352, 1443.

[7] a) H. Sadeghi, S. Sangtarash, C. Lambert, Nano Lett. 2017, 17, 4611-4618; b) C. G. Péterfalvi, C. J. Lambert, Phys. Rev. B 2012, 86, 085443.

[8] P. Gehring, A. Harzheim, J. Spiece, Y. Sheng, G. Rogers, C. Evangeli, A. Mishra, B. J. Robinson, K. Porfyrakis, J. H. Warner, O. V. Kolosov, G. A. D. Briggs, J. A. Mol, Nano Lett. 2017, 17, 7055-7061.

[9] E. Burzurí, J. O. Island, R. Díaz-Torres, A. Fursina, A. González-Campo, O. Roubeau, S. J. Teat, N. Aliaga-Alcalde, E. Ruiz, H. S. J. van der Zant, ACS Nano 2016, 10, 2521-2527.

[10] a) P. Gehring, J. K. Sowa, J. Cremers, Q. Wu, H. Sadeghi, Y. Sheng, J. H. Warner, C. J. Lambert, G. A. D. Briggs, J. A. Mol, ACS Nano 2017, 11, 5325-5331; b) C. S. Lau, H. Sadeghi, G. Rogers, S. Sangtarash, P. Dallas, K. Porfyrakis, J. Warner, C. J. Lambert, G. A. D. Briggs, J. A. Mol, Nano Lett. 2016, 16, 170-176.

[11] K. Ullmann, P. B. Coto, S. Leitherer, A. Molina-Ontoria, N. Martín, M. Thoss, H. B. Weber, Nano Lett. 2015, 15, 3512-3518.

[12] a) P. Moreno-García, M. Gulcur, D. Z. Manrique, T. Pope, W. Hong, V. Kaliginedi, C. Huang, A. S. Batsanov, M. R. Bryce, C. Lambert, T. Wandlowski, J. Am. Chem. Soc. 2013, 135, 12228-12240; b) W. Hong, D. Z. Manrique, P. Moreno-García, M. Gulcur, A. Mishchenko, C. J. Lambert, M. R. Bryce, T. Wandlowski, J. Am. Chem. Soc. 2012, 134, 2292-2304; c) F. Chen, X. Li, J. Hihath, Z. Huang, N. Tao, J. Am. Chem. Soc. 2006, 128, 15874-15881; d) E. Leary, A. La Rosa, M. T. Gonzalez, G. Rubio-Bollinger, N. Agrait, N. Martin, Chem. Soc. Rev. 2015, 44, 920-942.

[13] F. Prins, A. Barreiro, J. W. Ruitenberg, J. S. Seldenthuis, N. Aliaga-Alcalde, L. M. K. Vandersypen, H. S. J. van der Zant, Nano Lett. 2011, 11, 4607-4611.

[14] S. Lumetti, A. Candini, C. Godfrin, F. Balestro, W. Wernsdorfer, S. Klyatskaya, M. Ruben, M. Affronte, Dalton Trans. 2016, 45, 16570-16574.

[15] J. O. Island, A. Holovchenko, M. Koole, P. F. A. Alkemade, M. Menelaou, N. Aliaga-Alcalde, E. Burzurí, H. S. J. van der Zant, J. Phys.: Condens. Matter 2014, 26, 474205.

[16] a) P. Gehring, H. Sadeghi, S. Sangtarash, C. S. Lau, J. Liu, A. Ardavan, J. H. Warner, C. J. Lambert, G. A. D. Briggs, J. A. Mol, Nano Lett. 2016, 16, 4210-4216; b) A. Barreiro, H. S. J. van der Zant, L. M. K. Vandersypen, Nano Lett. 2012, 12, 6096-6100.

[17] a) J. A. Mann, W. R. Dichtel, ACS Nano 2013, 7, 7193-7199; b) J. K. Dutton, J. H. Knox, X. Radisson, H. J. Ritchie, R. Ramage, J. Chem. Soc. Perkin Trans. I 1995, 2581-2587.

[18] K. R. Paserba, A. J. Gellman, J. Chem. Phys. 2001, 115, 6737-6751.

[19] F. C. Grozema, C. Houarner-Rassin, P. Prins, L. D. A. Siebbeles, H. L. Anderson, J. Am. Chem. Soc. 2007, 129, 13370-13371.

[20] a) M. El Abbassi, L. Posa, P. Makk, C. Nef, K. Thodkar, A. Halbritter, M. Calame, Nanoscale 2017, 9, 17312-17317; b) C. Nef, L. Posa, P. Makk, W. Fu, A. Halbritter, C. Schonenberger, M. Calame, Nanoscale 2014, 6, 7249-7254; c) C. S. Lau, J. A. Mol, J. H. Warner, G. A. D. Briggs, Phys. Chem. Chem. Phys. 2014, 16, 20398-20401. 
[21] F. Prins, A. Barreiro, J. W. Ruitenberg, J. S. Seldenthuis, N. Aliaga-Alcalde, L. M. Vandersypen, H. S. van der Zant, Nano Lett. 2011, 11, 4607-4611.

[22] J. Moser, A. Barreiro, A. Bachtold, Appl. Phys. Lett. 2007, 91, 163513.

[23] a) J. G. Simmons, J. Appl. Phys. 1963, 34, 1793-1803; b) A. Mangin, A. Anthore, M. L. Della Rocca, E. Boulat, P. Lafarge, Phys. Rev. B 2009, 80, 235432.

[24] J. S. Cameron, D. S. Ashley, J. S. Andrew, G. S. Joseph, T. G. Christopher, Nanotechnology 2016, 27, 125704.

[25] V. M. García-Suárez, C. J. Lambert, Phys. Rev. B 2008, 78, 235412.

[26] The $p$-values calculated for molecules 5 and 6 are still below 0.05 even if the JFP is defined as (category A)/(categories $A+B+C)$, see SI.

[27] M. S. José, A. Emilio, D. G. Julian, G. Alberto, J. Javier, O. Pablo, S.-P. Daniel, J. Phys.: Condens. Matter 2002, 14, 2745.

[28] Z. Peng, R. Yang, M. A. Kim, L. Li, H. Liu, RSC Adv. 2017, 7, 27048-27057.

[29] C. W. J. Beenakker, Phys. Rev. B 1991, 44, 1646-1656.

[30] H. Sadeghi, J. A. Mol, C. S. Lau, G. A. D. Briggs, J. Warner, C. J. Lambert, Proc. Natl. Acad. Sci. U. S. A. 2015, 112, 2658.

[31] a) J. Ping, R. Vishnubhotla, A. Vrudhula, A. T. C. Johnson, ACS Nano 2016, 10, 8700-8704; b) J. Ping, R. Vishnubhotla, J. Xi, P. Ducos, J. G. Saven, R. Liu, A. T. C. Johnson, ACS Nano 2018, 12, 4218-4223; c) J. A. Mann, T. Alava, H. G. Craighead, W. R. Dichtel, Angew. Chem., Int. Ed. 2013, 52, 3177-3180; d) M. Zhang, R. R. Parajuli, D. Mastrogiovanni, B. Dai, P. Lo, W. Cheung, R. Brukh, P. L. Chiu, T. Zhou, Z. Liu, E. Garfunkel, H. He, Small 2010, 6, 1100-1107. 
Molecules can be interfaced with graphene electrodes using non-covalent interactions to generate single-molecule transistors. A family of pi-stacking anchor groups has been synthesized and the yield of transistors depends strongly on the nature of the anchor group. Whilst coupling to electrodes varies between devices, it is possible to identify junctions functionalized with single molecules if they exhibit characteristic addition energies.

Keyword: molecular electronics

B. Limburg, J. O. Thomas, G. Holloway, H. Sadeghi, S. Sangtarash, I. C.-Y. Hou, J. Cremers, A. Narita, K. Müllen, C. J. Lambert, G. A. D. Briggs, J. Mol, H. L. Anderson

\section{Anchor Groups for Graphene-Porphyrin Single-Molecule Transistors}

ToC figure:

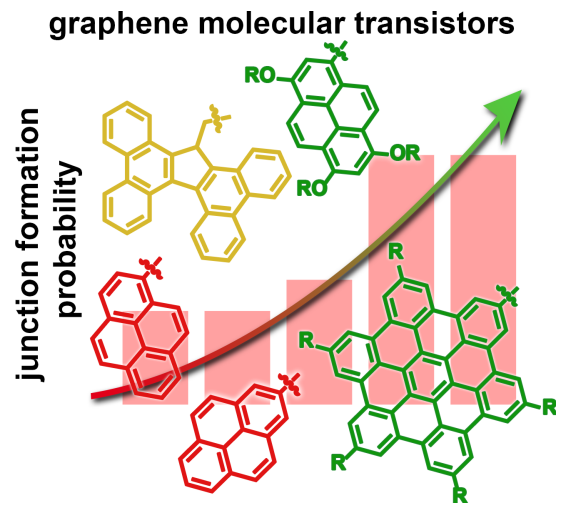

